Economía, Sociedad y Territorio, vol. viII, núm. 26, 2008, 381-413

\title{
Estrategias que favorecen u obstaculizan el desarrollo local en el marco de la globalización
}

\author{
José Alejandro Vargas-Castro* \\ Prudencio Óscar Mochi-AlemáN* *
}

\begin{abstract}
This paper analyses the strategies undertaken by local areas within the globalisation framework. We emphasise how some of these strategies can favour or hinder the ranking of the local area. We present three case studies in the State of Mexico: San Mateo Atenco, Valle de Bravo and Villa Guerrero. We identified some capacities of the intervening social players that allow a region to establish strategies that strengthen its local development. Nonetheless, the development is obstructed where no strategy is in place. The analysis of these capacities is carried out from three independent variables: organisational, instrumental and systemic capacities. We demonstrate that local development depends on these variables at international, national and local levels in a globalised context.
\end{abstract}

Keywords: globalisation, local development, citizen participation, political and economical planning.

\section{Resumen}

En este artículo se analizan las estrategias emprendidas desde los ámbitos locales en el marco de la globalización y se destaca cómo, si bien algunas de ellas favorecen su posicionamiento, otras lo obstaculizan. Se presentan tres casos de estudio en el Estado de México: San Mateo Atenco, Valle de Bravo y Villa Guerrero. Se logró identificar ciertas capacidades de los actores sociales que permiten recurrir a estrategias de concertación que favorecen el desarrollo local; sin embargo, ahí donde se carece de ellas el desarrollo se obstaculiza. El análisis de estas capacidades se hace a partir de tres variables independientes: capacidad organizacional, capacidad instrumental y capacidad sistémica. Se pretende demostrar cómo el desarrollo local depende de estas variables en los ámbitos internacional, nacional y local, en un contexto globalizado.

Palabras clave: globalización, desarrollo local, participación ciudadana, planeación política y económica.

"Universidad Autónoma del Estado de México. Correo-e: avargas@uaemex.mx, alejandro@siagem.com.mx.

**Universidad Nacional Autónoma de México. Correo-e: mochiprudencio@hotmail. com, pom@correo.crim.unam.mx. 


\section{Introducción}

En las últimas décadas los procesos de globalización alientan y acompañan importantes cambios estructurales de tipo económico, tecnológico, político, social y cultural. En realidad, todos estos cambios han puesto a prueba la capacidad de las economías, las instituciones y el conjunto de actores sociales para adaptarse a las nuevas circunstancias.

Si bien existe una gran variedad de aproximaciones y énfasis distintos sobre la globalización: integración funcional de actividades económicas internacionalmente dispersas (Gereffi, 1995); concentración del tiempo y del espacio (Harvey, 1995); articulación en tiempo real de actividades sociales localizadas en espacios geográficos diferentes (Castells, 1998); articulación directa de lo global y lo local en prácticas de lo glocal (Featherstone, 1990); rebasamiento del Estado nacional por las nuevas relaciones transnacionales o mundiales (Petrella, 1992; Beck, 1998; Dabat, 2000); mosaico global emergente de sistemas regionales de producción y cambio (Scout, 1998); sistematicidad de las nuevas interacciones (Axford, 1995) o nueva geoeconomía (Dicken, 1998). Como bien señala Dabat (2002: 66), tales diferencias no implican tantos puntos de vista excluyentes sobre la naturaleza del fenómeno, sino más bien, énfasis y jerarquizaciones distintas de aspectos diferentes de un mismo proceso complejo.

En este sentido ubicamos la globalización como nueva configuración espacial-territorial (Mochi y Girardo, 1998), haciendo énfasis no sólo en la variable espacial sino más específicamente en la territorial. El espacio se entiende como soporte geográfico donde se desenvuelven las actividades socioeconómicas -lo cual lleva implícita la idea de homogeneidad-como procesos que unificaron el mercado mundial, la liberalización comercial, el papel de las comunicaciones y las reducciones de los costos de transporte. La variable territorial, en cambio, incluye la heterogeneidad y complejidad del mundo real expresada en el territorio como actor del desarrollo.

En este contexto es importante destacar que muchos países de industrialización tardía (como India, Irlanda, Israel, Taiwán, China, Singapur, Tailandia, Corea, Filipinas, Vietnam, entre otros) tuvieron un papel destacado, alcanzando buenos niveles de crecimiento e inserción en los mercados internacionales. Estos nuevos escenarios planteados a partir de la nueva división global del trabajo (Gereffi, 1995), durante los años noventa también ofre- 
cen oportunidades a algunos países de América Latina (Brasil, Argentina, México, Costa Rica, Uruguay, Chile, Venezuela, entre otros) aunque de manera mucho más acotada y desigual. Muchas naciones y regiones no se pudieron incorporar a estas dinámicas y por ende se agudizaron aún más sus problemas económicos y sociales. Por ejemplo, políticas arancelarias en los países desarrollados impiden el acceso a sus mercados a productos decisivos en las exportaciones de países en desarrollo.

A pesar de ello, los retos impuestos por los cambios estructurales y la globalización conllevan conjuntamente a un contexto donde cobran mayor importancia los territorios. Dicho de otra manera, la competencia internacional introduce también de manera progresiva mayores exigencias y oportunidades a todos los espacios locales. Basta citar, por ejemplo, la existencia de sistemas productivos locales en una gran diversidad de países -de acuerdo con investigaciones realizadas durante los últimos años (Albuquerque, 2005, 2003, 1997; Madoery, 2001; Boisier, 2005; Vázquez Barquero, 2001; Vargas, 2006) - como Italia (Terza Italia), España (Comunidad Valenciana, Barcelona), Portugal (Val do Ave y región norte), Argentina (Rosario, Rafaela-Santa Fe), Brasil (Río Grande do Sul), México (Guanajuato, San Mateo Atenco, Valle de Bravo, Villa Guerrero, éstas tres últimas en el Estado de México), Pakistán (Sialkot), India (Tiruppur), entre muchos otros municipios y regiones. Diversas experiencias internacionales han pasado a ser referentes para otras intervenciones en ciudades o contextos locales de características similares. Si bien no se puede hablar de aplicar recetas estándar a realidades sociales que siempre son diferentes en algún grado, no es menos cierto que ya existe un cierto bagaje metodológico y teórico que podemos tomar como referencia.

Es posible señalar, entonces, que la adecuación de los sistemas económicos nacionales a este nuevo entorno de competitividad ha dado lugar a que en los ámbitos locales se generen, por un lado, condiciones de apertura y acceso a información, recursos y mercados internacionales para algunos productores locales y, por otra parte, una competencia franca en términos de calidad y precio que algunos productores locales no están en condiciones de afrontar.

En este contexto de retos y oportunidades, la respuesta de los ámbitos locales ha sido diferenciada en forma e intensidad, pues en algunos casos se observa la creación de condiciones que permiten una inserción competitiva en el ámbito internacional y, en 
otros, un retraimiento de los actores locales que, ante el embate del nuevo contexto global, ven fragmentada y vulnerada su organización económica y aun la social.

Así, cada territorio necesitará articularse con la globalización en función de su propia historia, de sus posibilidades específicas (perfil productivo, características medioambientales, problemática sociolaboral y cultural), así como de su capacidad para adaptarse a las exigencias de eficiencia productiva y competitividad no sólo en actividades industriales sino en el conjunto de la economía, bien en el ámbito rural o urbano, bien en los sectores agrario, minero o de servicios (Vázquez Barquero, 2005).

En el caso del Estado de México, y particularmente en los municipios que se toman como referente empírico en este artículo, se observan diferentes formas de afrontar esta situación, en la que destaca la capacidad de organización de los actores locales para definir cómo aprovechar o enfrentar las condiciones de un contexto caracterizado por la irrupción de factores o elementos de carácter internacional, convencionalmente relacionados con la globalización.

En este sentido cabe preguntarnos: ¿qué relación guardan los procesos globales con los locales? ¿Cómo se manifiestan estos procesos en el ámbito local? ¿Qué circunstancias podrían explicar el éxito de algunos territorios y el estancamiento de otros? ¿Cuáles estrategias de los actores locales pueden favorecer u obstaculizar el desarrollo local?

Con la finalidad de tener referentes empíricos para analizar las relaciones que implican estas interrogantes, se consideran tres casos de estudio ubicados en el Estado de México, la entidad federativa con mayor población del país, que además se distingue por la heterogeneidad de su composición social y económica. Se trata de tres municipios pequeños con una población de alrededor de sesenta mil habitantes.

Se consideró a San Mateo Atenco, cuya producción de calzado sufre fuertes presiones por la introducción de zapatos de procedencia oriental y con una respuesta desarticulada por parte de los actores locales; Valle de Bravo, que se distingue por su vocación turística, la riqueza de sus recursos naturales, el valor relativo y relacional de su territorio, así como la confrontación de objetivos, posiciones y proyectos por parte de los actores locales; y Villa Guerrero, por la consolidación de la organización e iniciativas de los actores locales y el posicionamiento de su producción florícola en el ámbito internacional. Estos tres munici- 
pios muestran un cierto dinamismo económico y, sobre todo, una compleja relación entre los actores locales. El desafío que nos propusimos con esta investigación es precisamente identificar qué tipo de estrategias de los actores locales pueden favorecer $\mathrm{u}$ obstaculizar el desarrollo local en el marco de la globalización.

\section{Las peculiaridades que distinguen la relación entre lo global y lo local}

En la producción teórica sobre el desarrollo local se hace referencia de manera constante a las implicaciones del proceso de globalización desde diferentes perspectivas, las cuales tienen consideraciones que resultan divergentes en relación con los efectos que la globalización genera en los ámbitos locales.

Una de las perspectivas señaladas estriba en considerar la globalización como un proceso que genera las condiciones propicias para impulsar el desarrollo local, en función de la posibilidad de explorar nuevas opciones para comercializar los productos y servicios locales, acceder a información, tecnología y formas novedosas de organizar la producción, así como para incrementar la competitividad del territorio, como estrategia para atraer la inversión extranjera (OCDE, 2002).

Por otra parte, algunos autores enfatizan los aspectos de ese proceso que desarticulan y avasallan a las sociedades y economías locales, planteando que ante el incremento de la competencia en los mercados internacionales, los requerimientos de mayor apertura de las fronteras nacionales y la eliminación gradual de restricciones a los flujos de mercancías y dinero entre países, la capacidad de respuesta de algunas comunidades es limitada, lo que origina fuertes cambios en la organización social y económica de las mismas (Vázquez Barquero, 2001).

Los planteamientos anteriores no indican que los autores referidos se ubiquen necesariamente a favor de una o otra postura; generalmente se reconoce que para las sociedades locales el proceso de globalización representa retos y oportunidades, sin embargo, según su enfoque, enfatizan alguna de las dos posibilidades.

Es importante destacar, como se señaló en la Introducción, que asumimos que la globalización implica cambios sociales, culturales, territoriales e, incluso, ideológicos en los ámbitos locales; sin embargo, existe consenso sobre la importancia que tienen los procesos de carácter económico, lo que se puede explicar si consideramos que es precisamente en este aspecto donde se 
pueden identificar con mayor claridad los vínculos entre ambas facetas de la realidad: lo local y lo global.

Dentro de las dimensiones que nos permiten identificar de qué manera se manifiesta la globalización en lo local, podemos sintetizar algunas de sus peculiaridades más sobresalientes:

\section{El paradigma del desarrollo endógeno}

Los procesos corporativos de integración vertical que signaron el siglo Xx se caracterizaron por estructuras de representación corporativas centradas en el Estado-nación donde predominaron sujetos como burocracias estatales, organizaciones empresariales, élites modernizantes, vanguardias iluminadas, actores privados corporativos, entre otros. Esto tuvo su correlato en las disciplinas sociales que analizaban los procesos económicos, políticos y sociales desligados del territorio. Los municipios no intervenían en las estrategias centralizadas del desarrollo, durante décadas desarrollo y territorio tuvieron un punto de encuentro exclusivamente nacional (Madoery, 2001). En los últimos años, sin embargo, gran parte de los actores y disciplinas sociales parten de la dirección opuesta, o sea, de la perspectiva de los ambientes locales. La atención se centra en el desarrollo de los territorios -particularmente regiones-, las localidades, las ciudades y las metrópolis para identificar las características de su desarrollo basado en su identidad, su historia y en sus recursos materiales e inmateriales. En este sentido, los procesos endógenos de los territorios cobran especial relevancia para este nuevo enfoque, el cual considera que las economías de las regiones y las localidades pueden crecer utilizando el potencial de desarrollo existente en el territorio.

El desarrollo es endógeno debido a su estrecha asociación con la cultura local y con los valores que ella incluye. Desarrollo endógeno significa, en efecto, la capacidad para transformar el sistema socioeconómico, la habilidad para reaccionar ante los desafíos externos, la promoción del aprendizaje social y el talento para introducir formas específicas de regulación social en el ámbito local que favorecen el desarrollo de las características anteriores. En otras palabras, desarrollo endógeno es la habilidad para innovar en el ámbito local (Garófoli, 1995).

Esta posición del desarrollo constituye un modelo alternativo al propuesto por el paradigma del desarrollo exógeno (en boga durante los años cincuenta y sesenta), según el cual el crecimien- 
to económico era producto de los procesos de industrialización y concentración de la actividad productiva -a través de grandes plantas-, en unos cuantos centros urbanos grandes.

La novedad de estos procesos consiste en que ya no cuentan sólo las elecciones derivadas del centro -de políticas nacionales centralizadas del Estado- o determinantes espaciales -como la dotación de particulares recursos naturales y ambientales- o la cercanía con algunos mercados, sino que el elemento determinante en el nuevo contexto es el protagonismo de actores organizados y de las instituciones locales que desarrollan experiencias de cooperación y concertación innovadora mediante la construcción de acuerdos que involucran y favorecen al conjunto de actores en el territorio.

Adicionalmente, el desarrollo endógeno puede plantearse como una interpretación de la interacción entre tecnología, organización de la producción, desarrollo urbano e instituciones en la dinámica económica que permite proponer medidas para estimular los procesos de acumulación de capital a partir de aprovechar el potencial de desarrollo endógeno, constituido por los recursos naturales, humanos, históricos, tecnológicos y culturales de que dispone un territorio.

Articulación entre lo global, lo nacional, lo regional y lo local

Otra peculiaridad evidente en la relación global-local es que los modelos de desarrollo local no tienen que ver con un localismo autorreferencial ni con un regreso al rol de las economías autárquicas del medioevo, más bien se trata de la capacidad para articular y gestionar las políticas globales con las nacionales y las subnacionales.

El protagonismo de los actores locales se lleva a cabo considerando los niveles nacionales, macrorregionales o globales, atrayendo de manera inteligente recursos externos bien de tipo político (inversiones públicas calificadas o recursos para atraer empresas privadas), económico o cultural (vinculados con decisiones de inversiones o de localización de actores privados).

Entre los elementos de desarrollo local identificados con claridad en la literatura especializada destacan la disponibilidad de mano de obra calificada -o la posibilidad de formarla-, la existencia o viabilidad de construir redes de solidaridad e intercambio de conocimiento, dependencias gubernamentales que apoyen a las nuevas empresas y factores como el liderazgo de algunos 
actores, tradición participativa y conocimiento del mercado de trabajo local y global.

Cuando se logran captar las oportunidades que la extensión de los mercados ofrece, se potencian nuevas estrategias de producción de bienes y servicios que valoran competencias específicas y bienes comunes (como el patrimonio ambiental e históricoartístico). Desde esta perspectiva, el desarrollo local implica identificar en la globalización posibles oportunidades de desarrollo y adecuar sus estrategias para corresponder a las exigencias de la misma.

No se trata, por tanto, de resistir la globalización sino de rechazar los elementos nocivos de los procesos globales en el ámbito local y al mismo tiempo usar de manera inteligente -es decir, con capacidad de proyectar- las mayores y mejores oportunidades potenciales que los diversos niveles territoriales (globales, nacionales, macrorregionales) ofrece del lado de los procesos productivos más flexibles y con salidas diferentes hacia mercados locales, nacionales y globales.

Asimismo, el Estado nación no desaparece, como lo auguran las posiciones más conservadoras, sino que se reconfigura en función de estas nuevas necesidades de cambios estructurales. Quedan bajo su predominio los controles macroeconómicos (inflación, déficit público, balanza de pagos) así como otras funciones que debe garantizar (salud, educación, vivienda, etc.). Las administraciones subnacionales se articulan con la administración central pero bajo otro protagonismo, que les confiere que puedan atender problemas dentro del territorio.

\section{Valorización de los recursos locales}

Otro elemento distintivo en el marco de la globalización del desarrollo local es su capacidad para atraer recursos externos que tengan efecto en el territorio. Esta dinámica consiste en la capacidad de los territorios para atraer recursos externos para valorizar los internos: inversiones, empresas, recursos científicos y culturales no sólo como ocasión para el crecimiento de la producción, del rédito y de la ocupación, sino como instrumento que enriquece las competencias y las especializaciones locales.

Es fácil y recurrente confundir desarrollo local con crecimiento o dinamismo local, incluso, muchas veces es difícil de diferenciar ya que en una fase inicial los dos fenómenos pueden convivir. Los procesos de beneficios fiscales, financieros, niveles educati- 
vos, políticas de formación, costos del trabajo, servicios e infraestructura de un territorio deben servir para atraer inversiones siempre y cuando se combinen con la posibilidad de valorizar los recursos y capacidades locales. Si estos procesos no se dan de manera integral en el contexto actual de la globalización y en la redefinición e interacción entre distintos espacios y territorios, se corre el peligro de que no se genere un verdadero desarrollo local.

La competencia es cada vez más grande entre territorios y no entre empresas (Albuquerque, 2003) e implica que si un territorio no brinda la combinación justa para los flujos de capital, las empresas emigran o otros lugares donde les permitan generar más réditos. Muchos sitios sufren una inestabilidad fuerte y continuamente están sometidos a la competitividad de otros lugares y oportunidades (Coraggio, 2005). En este sentido, no cualquier territorio sirve a este objetivo ya que el desarrollo local se puede concebir sólo en los contextos donde las capacidades de cooperación y de concertación estratégica de los actores locales sirvan a la construcción de proyectos comunes. No se trata de basar la competitividad en la desregulación del medioambiente, en mano de obra a bajo costo, con baja calificación, sin derechos establecidos o subsidiando inversiones que no aportan mejoras a las comunidades locales.

Desde esta perspectiva, la búsqueda del desarrollo local surge en el contexto de la globalización como una respuesta de los actores locales para contener los efectos de la absorción mecánica de las economías y sociedades locales por un proceso amorfo y utilitario, y como una estrategia que revaloriza el potencial de los propios actores locales como agentes activos en la búsqueda de mejores condiciones de vida para su población, así como en dinamizadores de la economía local como palanca del desarrollo.

Los actores locales y sus iniciativas han permeado el proceso de globalización y ahora resurgen -como lo evidencia la prolífica literatura que aborda las experiencias del desarrollo local- con fuerza y mayores elementos de cohesión que les permiten incidir paulatinamente en los procesos de cambio de las relaciones institucionales, así como para dar paso a nuevas formas de relación entre sociedad y gobierno en la promoción del desarrollo local.

En este sentido, la globalización puede jugar un papel fundamental para incorporar nueva vitalidad a los agentes locales, quienes ahora encuentran formas novedosas de interrelacionarse, gracias al avance de la tecnología en comunicaciones y transportes, para de este modo formar redes de solidaridad y apoyo, así 
como nichos particulares de consumo para productos de carácter endémico, aprovechando procesos como la estandarización del consumo y la personalización de las preferencias, pues de lo que se trata más bien es de aprovechar las oportunidades de la globalización teniendo en cuenta la competitividad de la economía local, la democratización, la gobernabilidad y la sustentabilidad medioambiental.

\section{La importancia que adquiere el conocimiento y los nuevos procesos de innovación}

En esta nueva fase del desarrollo el conocimiento adquiere un valor fundamental, así como la articulación con el sector científico-educativo y el conjunto de la producción y los servicios sociales. En este marco, el concepto de competitividad como fenómeno sistémico ya no tiene que ver con los factores macroeconómicos y sectoriales tradicionales, sino con la conducta de los agentes, la creación de redes y con el grado de desarrollo del medio local (entornos innovadores).

En el marco de los procesos de innovación concebidos como desarrollo interactivo de aprendizaje en el que se introducen nuevos conocimientos o se combinan otros ya existentes para generar nuevas competencias (Lundvall, 1992; Gregersen y Johnson, 1996), el papel del entorno local y de sus instituciones en el desarrollo de la capacidad innovadora de las empresas adquiere nueva significación. La creciente importancia del conocimiento tácito, ${ }^{1}$ fuertemente anclado al territorio, resulta cada vez más relevante para absorber el conocimiento codificado. ${ }^{2}$ La innovación, en particular, parece fuertemente ligada a las interacciones

\footnotetext{
${ }^{1}$ La idea de conocimiento tácito la introdujo Polanyi, quien sostiene que "se conoce más de los que se puede decir" (1966). El concepto lo retomaron otros autores quienes señalan que "conocer tácitamente significa conocer sin distancia de las cosas y los actos, y la interacción cognoscitiva entre las personas se caracteriza porque es inconsciente" (Nonaka y Takeuchi (1995: 61-74). En nuestros términos, se define tácito el conocimiento difícil de definir y de expresar de manera formalizada porque está vinculado con el contexto de referencia y tiene una matriz profundamente personal.

${ }^{2}$ Conocimientos codificados: también llamados conocimientos explícitos para indicar esa parte del conocimiento que puede ser codificado, trasmitido y usado en contextos diversos de los que fue generado, como el conocimiento encerrado en un manual de instrucciones. Se trata de un conocimiento claro, determinado, de inmediata comprensión, simple de recoger y de mucho valor para las organizaciones, porque es fácil de guardar y difundir (Zack, 1999). Sin embargo no siempre al interior de una organización el conocimiento explícito está realmente codificado en documentos formales o con un lenguaje compartido, sino que por el contrario muchas veces ese conocimiento se mantiene implícitamente y compartido de manera informal.
} 
específicas entre agentes y a los conocimientos tácitos que los unen (Veltz, citado por Boisier, 2005).

En este nuevo escenario internacional, ambiente local y economía global no son términos antagónicos, ya que la globalización debe su fuerza a la complejidad del conocimiento y de la sinergia que se produce a partir de la confrontación competitiva de diferentes variantes territoriales y redes de agentes (Yoguel, 2005).

En este sentido, el desarrollo económico y la dinámica productiva dependen de la introducción de innovaciones (en los productos, en los procesos y servicios, en nuevos estilos de dirección y gestión empresarial, en las relaciones interempresariales, en la gestión pública, en la concertación de actores, etc.) que impulsen la transformación del sistema productivo y social del lugar. Para que ello sea posible, es necesario que los actores que forman parte del entorno tomen decisiones adecuadas de inversión, tecnológicas y organizativas. En esta capacidad reside la característica de innovación actual.

La capacidad de aprendizaje de los agentes locales, en un entorno que se transforma, orienta las decisiones de inversión y, por tanto, la respuesta de los sistemas locales a los desafíos de la competencia (Maillat, citado por Vázquez Barquero, 2005: 39).

Este proceso de innovación es posible gracias a las relaciones formales e informales entre actores públicos y privados (asociatividad), y la competitividad de estos entornos territoriales se logra cuando se neutralizan los costos de transacción producto de las redes entre empresas, centros de investigación, laboratorios tecnológicos, entre otros. Si la innovación en las empresas no va acompañada de una innovación difusa en el territorio, no se generarán modificaciones cualitativas que dinamicen todo el sistema en su conjunto.

La formación de redes (otro eje fundamental del desarrollo local en su vínculo con la globalización) permite el intercambio de productos, el flujo de información, la circulación de conocimientos, la transmisión de datos y las pautas de comportamiento que propician la difusión de las innovaciones, aumentan la productividad y mejoran la competitividad. El dinamismo del entorno local dependerá fundamentalmente de la densidad de estas redes.

\section{Capacidades y estrategias de los actores locales}

Ante los nuevos escenarios descritos, signados por los cambios estructurales y el nuevo dinamismo que adquieren los territo- 
rios, la institucionalidad local adquiere un papel central en el desarrollo de las personas, las sociedades y las ciudades, es decir, en la búsqueda del desarrollo local. Entendido éste como resultado de la conjugación de las capacidades de los individuos para articular sus iniciativas y diseñar instrumentos que les permitan mejorar su calidad de vida, según sus propias percepciones, lo cual se encuentra determinado precisamente por el marco institucional en el que se desenvuelven los actores locales.

Dicho marco se integra convencionalmente por las normas y reglamentaciones sociales que condicionan y orientan las acciones y decisiones de los individuos, así como por las características de su entorno, al cual se agrega, desde esta perspectiva, una serie de condicionantes exógenas derivadas del proceso de globalización, cuya consideración resulta insoslayable en el concepto integral del desarrollo local en el contexto global.

Las capacidades de los actores locales a que se hace referencia se constituyen por sus posibilidades de acción, habilidades y recursos en función de tres vertientes: la organización en torno a un objetivo común, de mejoramiento permanente de las condiciones de vida de la sociedad local; la generación de instrumentos adecuados y útiles para conseguir el objetivo señalado; y el conocimiento y visión de la posición que mantiene el ámbito local en un contexto más amplio, el de la globalización. En este sentido, para analizar los procesos de desarrollo local en los estudios de caso referidos, se identificaron tres capacidades fundamentales de los actores locales, a las que se denominó organizacional, instrumental y sistémica (Vargas, 2006).

La articulación de iniciativas corresponde a la capacidad organizacional y se refiere a las habilidades, posibilidades de acción y recursos que utilizan los actores locales para organizarse en torno a un objetivo común, de transformación cualitativa y sostenida de las condiciones de vida de una sociedad local.

Desde una perspectiva compatible con los postulados de Amartya Sen (2000), esta capacidad implica la aptitud de los actores para transformar sus recursos en instrumentos para promover el mejoramiento de su calidad de vida y expectativas de progreso, en este caso, tomando como base su organización y la formulación de un proyecto explícito de desarrollo local.

La capacidad instrumental corresponde a las habilidades y posibilidades de los actores locales para generar los instrumentos concretos que les permitan conseguir los objetivos de desarrollo local que pretenden, así como realizar las acciones especí- 
ficas con la finalidad de afrontar cambios en su entorno, manifestada por la existencia de instrumentos de planeación, concertación, gestión y de comunicación social.

La tercera capacidad analizada es la sistémica, que alude al conocimiento e interpretación que hacen los actores locales del complejo esquema de relaciones y factores exógenos que inciden en la configuración de la sociedad y la economía local, e incluye los ámbitos regional, estatal, nacional e internacional y, puede inferirse adicionalmente, por la existencia de vínculos específicos con actores externos, así como por el acceso y uso de las nuevas tecnologías de información y comunicación para apoyar sus iniciativas o proyectos de desarrollo.

Este tipo de capacidades está en función de las cualidades de agentes específicos, del conocimiento que tengan de la lógica de actuación de los actores involucrados, así como del marco institucional que les rige y del contexto nacional e internacional en que se insertan. En los casos de estudio analizados en este artículo, estas capacidades pueden considerarse determinantes.

Desde esta perspectiva, el desarrollo local se entiende como un proceso multidimensional, de formación y aprovechamiento de las capacidades de los actores locales y de los recursos disponibles con el objetivo de mejorar la calidad de vida de la población y de ampliar sus expectativas de progreso.

Se trata de un cambio estructural en los sistemas locales originado por la articulación de las iniciativas de los actores locales en torno a un factor de cohesión interna, que es la actividad económica fundamental de la localidad. Para ello diseñan estrategias, crean y utilizan diversos instrumentos e incluyen en su formulación la visión y percepción que tienen acerca de factores de índole internacional, cuya incidencia en el ámbito local se manifiesta de diferentes maneras para dar lugar a una serie de estrategias específicas.

\section{Análisis de los casos de estudio}

A partir del esquema de capacidades de los actores locales referido en párrafos anteriores, es importante señalar que en nuestra investigación de campo logramos recabar información que permitió caracterizar las diferentes respuestas y estrategias que implementaron los actores locales ante las manifestaciones de la globalización, donde se destaca el papel tan importante de la concertación entre agentes y la articulación de sus lógicas e ini- 
ciativas para impulsar un proyecto común, o bien, para afrontar condiciones adversas que modifican los equilibrios precarios en términos sociales, económicos y políticos en el territorio.

\subsection{San Mateo Atenco}

El municipio de San Mateo Atenco tiene una población de alrededor de 60,000 habitantes y se ubica en el centro del Estado de México. Forma parte de la Zona Metropolitana de la Ciudad de Toluca y su localización resulta privilegiada para el desarrollo de la actividad económica que le caracteriza: la producción y comercialización de calzado. Su ubicación tiene muchas ventajas ya que se encuentra relativamente cerca de la capital del país y de la ciudad de Toluca, capital del Estado de México y sitio en el que se concentran las principales unidades administrativas estatales.

Lo anterior le proporciona acceso directo a infraestructura y equipamiento regional de primer orden, como autopistas, aeropuertos, centros comerciales y expendios de materias primas e insumos secundarios para la elaboración del calzado, elementos de gran importancia para la principal actividad productiva del municipio y, de manera especial, para la comercialización del calzado.

En términos socioeconómicos, con base en información del Consejo Estatal de Población del Estado de México, San Mateo Atenco tiene un grado de marginación bajo, con un índice de -0.6792, determinado según los indicadores del cuadro 1.

Por su parte, el Instituto Nacional para el Federalismo y el Desarrollo Municipal establece en el Sistema Nacional de Información Municipal que San Mateo Atenco tiene un grado de marginación muy bajo y presenta cifras que evidencian la disminución gradual en los indicadores considerados ${ }^{3}$ (cuadro 2).

Esta misma institución establece que en el año 2000 San Mateo Atenco tenía un nivel de desarrollo humano medio alto, estimado en un puntaje de 0.776 , que incluye $94.6 \%$ de personas mayores de 15 años alfabetas; 61.7\% de personas entre 6 y 24 años que asistían a la escuela y un producto interno bruto (PIB) de 4,353 dólares entre sus principales indicadores.

De la caracterización territorial y socioeconómica de San Mateo Atenco se desprenden las siguientes consideraciones:

${ }^{3}$ Porcentajes de población analfabeta de 15 años o más; sin primaria completa; en localidades con menos de 5,000 habitantes; de población con ingresos de hasta dos salarios mínimos; de viviendas sin drenaje ni excusado, sin energía eléctrica, sin agua entubada, con hacinamiento y con piso de tierra. 
Cuadro 1

Indicadores de marginación, 2002

\begin{tabular}{lc}
\hline \multicolumn{1}{c}{ Indicadores } & $\begin{array}{c}\text { San Mateo Atenco } \\
\text { (porcentaje) }\end{array}$ \\
\hline Población & 59,647 \\
Población analfabeta mayor de 16 años & 5.87 \\
Población sin primaria completa mayor de 16 años & 17.20 \\
Ocupantes de viviendas sin drenaje ni excusado & 4.77 \\
Ocupantes de viviendas sin energía eléctrica & 1.45 \\
Ocupantes de viviendas sin agua entubada & 24.21 \\
Viviendas con hacinamiento & 54.28 \\
Ocupantes de viviendas con piso de tierra & 5.98 \\
Población en localidades con menos de & 3.70 \\
6,000 habitantes & \\
Población ocupada con ingreso menor a dos & 51.87 \\
salarios mínimos & -0.6792 \\
Índice de marginación & Baja \\
Grado &
\end{tabular}

Fuente: Consejo Estatal de Población, Estado de México, Índices de marginación 2000. 124 municipios, 2002.

\section{Cuadro 2}

Comparativo del índice y grado de marginación, 1980-2000

\begin{tabular}{lrrrr}
\hline Año/aspecto a considerar & 1980 & 1990 & 1995 & 2000 \\
\hline Índice de marginación & -18.41 & -1.314 & -1.195 & -1.356 \\
Grado de marginación & Media & Baja & Baja & Muy baja \\
Posición en el contexto nacional & 2,267 & 2,158 & & 2,238 \\
\hline
\end{tabular}

Fuente: Instituto Nacional para el Federalismo y el Desarrollo Municipal, Sistema Nacional de Información Municipal.

- El municipio cuenta con una serie de ventajas de ubicación que representan activos importantes para el desarrollo de la actividad económica predominante en el municipio, entre las que destacan su cercanía con los principales mercados nacionales y regionales, centros de investigación, diseño y comercialización, así como el acceso a infraestructura, equipamiento y servicios.

- Sus características socioeconómicas permiten inferir una dinámica importante, fundamentalmente en términos comparativos respecto de los indicadores estatales en cuanto al nivel de empleo, ingresos, así como de personal ocupado por unidad económica censable. 
- No obstante lo anterior, y como se plantea posteriormente, ante la introducción de calzado proveniente de China y de León (Guanajuato), en México, los actores locales se encuentran en franca desventaja y con pocas posibilidades de competir de manera exitosa, con base en las características de sus capacidades organizacional, instrumental y sistémica.

La importancia de la producción de calzado en San Mateo Atenco se aprecia con claridad si se considera que, de acuerdo con Francisco Torres (2002: 47), de las 133 empresas productoras en el año 2000 en el Estado de México, 72.93\% se localizan en este municipio, teniendo como característica principal que $81.95 \%$ de ellas se consideran microempresas, es decir, que tienen entre uno y nueve trabajadores.

En la actualidad, la industria manufacturera -en la que se inscribe la producción de calzado- ocupa más de $50 \%$ de la población económicamente activa (PEA) y en el sector terciario -en el que se ubica la comercialización y prestación de servicios a productores y consumidores de calzado- se ocupa 43\% aproximadamente.

Como se puede observar en el cuadro 3, la distribución porcentual de la PEA disminuyó drásticamente en el caso de las actividades agrícolas, ganaderas y lacustres en el municipio, para acumularse de forma mayoritaria en la producción de manufacturas y en actividades comerciales y de prestación de servicios.

Cabe destacar el incremento que ha tenido el sector terciario en relación con el comercio, actividad que complementa la producción del calzado. Aunque también se registra un decremento de aproximadamente seis puntos porcentuales en la PEA dedicada al sector secundario. Una posible explicación de este hecho estriba en que, de acuerdo con las personas entrevistadas, muchos de los pequeños productores han encontrado más redituable dedicarse al comercio de calzado chino o de León (Guanajuato) que a la producción en pequeña escala.

Si bien el desplazamiento de los productos locales por otros de procedencia externa dio lugar, en fechas recientes, a la formación de una organización general de los productores locales que pretende articular las iniciativas específicas existentes, es persistente la preeminencia de sus intereses particulares ante los de la sociedad municipal en su conjunto, dado que se mantiene 
Cuadro 3

Distribución porcentual de la PEA, 1970-2000

\begin{tabular}{lrrr}
\hline Sector económico & \multicolumn{3}{c}{ Porcentaje del total } \\
& $1970 *$ & 1990 & 2000 \\
\hline Primario & 27.94 & 4.66 & 1.69 \\
Secundario & 41.13 & 57.75 & 51.78 \\
Terciario & 24.15 & 35.21 & 43.41 \\
No especificado & 6.78 & 2.38 & 3.11 \\
Total & 100 & 100 & 100 \\
\hline
\end{tabular}

*Datos referentes a 1969.

Fuente: SIC-INEGI, 1972-2001: IX-XII Censos Generales de Población y Vivienda 19702000, resultados definitivos.

como principal objetivo para cada uno de ellos el mismo nicho de mercado.

La focalización de la producción hacia el mercado interno exclusivamente, así como la competencia que esto genera entre los productores locales, impide el intercambio de técnicas, tecnología y diseños que podrían incrementar la calidad y mejorar el precio de sus productos, que aunado al desconocimiento, o el soslayamiento, de la importancia de las características funcionales y estructurales del contexto internacional para la producción y comercialización del calzado, evidencian también la baja capacidad instrumental y sistémica de los actores locales en los términos en que éstas se han definido.

Esta situación ha generado diversos efectos en la organización social y económica del municipio, representados fundamentalmente por la transferencia de recursos entre los sectores convencionales de la economía local, lo que da lugar a un incremento en las actividades comerciales en detrimento de las manufactureras, pues cada vez es más común observar a pequeños productores que cambian su actividad económica, es decir, dejan de fabricar zapatos en sus propios talleres para dedicarse a vender o revender calzado foráneo.

Si bien la evolución de la fabricación de zapatos, como factor de cohesión interna, ha congregado esfuerzos y recursos de los productores locales -que por otra parte son los principales actores del desarrollo local-, la forma en que se presenta tal congregación no corresponde con un ejercicio de complementariedad o coordinación que redunde en beneficio de los propios productores locales, pues se trata sobre todo de la organización de diferentes asociaciones de un mismo gremio cuyo objetivo funda- 
mental es la competencia por el mercado interno, en términos de la producción local, más que su fortalecimiento para afrontar la competencia del calzado foráneo.

Lo anterior evidencia los efectos devastadores de la irrupción de este tipo de productos, que junto con la poca capacidad de respuesta de los productores locales y su baja competitividad, permite suponer que en breve tendrán que enfrentar no sólo la competencia que representa el volumen y calidad de los productos foráneos que invaden el mercado local, sino que en lo sucesivo se encontrarán ante el desafío de nuevos estándares de calidad y precio, para ajustarse a las especificidades de la demanda de los consumidores.

De este modo, la existencia de condicionantes socioculturales, institucionales y las provenientes del contexto de la globalización dan como resultado una baja capacidad organizacional en San Mateo Atenco, que se manifiesta también en la carencia de un proyecto concreto de desarrollo local.

\subsection{Valle de Bravo}

Este municipio se localiza en la zona surponiente del Estado de México, a una distancia aproximada de $130 \mathrm{~km}$ del Distrito Federal y a $75 \mathrm{~km}$ de la ciudad de Toluca. Tiene una población estimada en más de 60,000 habitantes. Se distingue por sus atractivos turísticos, principalmente la combinación de bosques y una presa artificial, que generan el interés especial de varias empresas inmobiliarias.

Es importante señalar el incremento poblacional que tuvo lugar en la década de 1990-2000, años en los que pasó de 36,135 a 57,375 habitantes, es decir, 21,240 nuevos pobladores que representan un aumento de $37 \%$ en tan corto tiempo, situación cuyo impacto social, territorial y, sobre todo, ambiental ha tenido resultados dramáticos que en las posibilidades de desarrollo local de Valle de Bravo deben valorarse con detenimiento, pues como se señaló, es precisamente la combinación de su belleza paisajística, recursos naturales y arquitectura vernácula lo que ha dado lugar al auge económico sostenido de este municipio; y son justo estos recursos los que peligran por la forma que asume actualmente el desarrollo urbano.

En términos socioeconómicos, el Instituto Nacional para el Federalismo y el Desarrollo Municipal establece que en 1980 el grado de marginación era medio, sin embargo, a partir de los años noventa éste se considera bajo, como se observa en el cua- 
Cuadro 4

Comparativo del índice y grado de marginación, 1980-2000

\begin{tabular}{lrrrr}
\hline Año/aspecto a considerar & 1980 & 1990 & 1995 & 2000 \\
\hline Índice de marginación & -13.540 & -0.873 & -0.909 & -0.733 \\
Grado de marginación & Media & Baja & Baja & Baja \\
Posición en el contexto nacional & 2,161 & 1,895 & & 1,802 \\
\hline
\end{tabular}

Fuente: Instituto Nacional para el Federalismo y el Desarrollo Municipal, Sistema Nacional de Información Municipal.

Cuadro 5

Indicadores de marginación, 2002

\begin{tabular}{lr}
\hline \multicolumn{1}{c}{ Indicadores } & $\begin{array}{r}\text { Valle de Bravo } \\
\text { (porcentaje) }\end{array}$ \\
\hline Población & 57,375 \\
Población analfabeta mayor de 16 años & 11.95 \\
Población sin primaria completa mayor de 16 años & 36.29 \\
Ocupantes de viviendas sin drenaje ni excusado & 18.06 \\
Ocupantes de viviendas sin energía eléctrica & 6.12 \\
Ocupantes de viviendas sin agua entubada & 7.54 \\
Viviendas con hacinamiento & 52.66 \\
Ocupantes de viviendas con piso de tierra & 15.30 \\
Población en localidades con menos de 6,000 habitantes & 44.37 \\
Población ocupada con ingreso menor a dos salarios mínimos. & 55.95 \\
Índice de marginación & 0.0238 \\
Grado & Medio \\
\hline
\end{tabular}

Fuente: Consejo Estatal de Población, Estado de México, Índices de marginación 2000. 124 municipios, 2002.

dro 4, lo que permite inferir el mejoramiento paulatino de las condiciones de vida de su población.

Por su parte, el Consejo Estatal de Población del Estado de México establece en su índice de marginación 2000 para los municipios del Estado de México, que Valle de Bravo presenta un grado de marginación medio, sustentado en la valoración de los indicadores que se muestran en el cuadro 5, que evidencian la polarización de los niveles de vida que se observan en el municipio.

En relación con el índice de desarrollo humano de este municipio, el Instituto Nacional para el Federalismo y el Desarrollo Municipal establece que es de 0.738 , lo que se considera como medio alto.

De la caracterización territorial y socioeconómica de Valle de Bravo se desprenden las siguientes consideraciones: 


\section{Cuadro 6}

PEA, 1970-2000

\begin{tabular}{lrrr}
\hline \multicolumn{1}{c}{$\begin{array}{c}\text { Sector } \\
\text { económico }\end{array}$} & \multicolumn{3}{c}{ Año-habitantes de 12 años y más } \\
& 1970 & 1990 & 2000 \\
\hline Primario & 2,214 & 2,174 & 1,717 \\
Secundario & 1,609 & 3,205 & 4,622 \\
Terciario & 1,318 & 4,346 & 9,901 \\
No especificado & 686 & 732 & 632 \\
Total & 5,827 & 10,457 & 16,872 \\
\hline
\end{tabular}

Fuente: SIC-INEGI, 1972-2001: IX-XII Censos Generales de Población y Vivienda 19702000, resultados definitivos.

- El municipio cuenta con una serie de elementos que lo posicionan como el principal destino turístico del Estado de México. Esta situación genera una dinámica económica intensa, caracterizada por el predominio de las actividades propias del sector terciario, fundamentalmente el que se relaciona con el comercio y la prestación de servicios al turismo.

- La industria de la construcción se observa como la segunda actividad económica en cuanto a la distribución de la PEA municipal, lo que genera una relación de complementariedad ya que la mayor parte de las edificaciones del municipio corresponde a casas de fin de semana, que constituyen el alojamiento de quienes se catalogan como turismo residente.

En el cuadro 6 se aprecia la evolución de la PEA del municipio, lo que nos permite conocer el comportamiento de los tres sectores convencionales que denotan las variaciones más importantes en los sectores primario y terciario: el primero presenta una fuerte tendencia decreciente, mientras que en el caso del sector terciario, su incremento es de tal magnitud que para el año 2000 representa más del doble de la registrada en la década anterior.

Si bien los indicadores cuantitativos del municipio reflejan una economía dinámica y con amplias posibilidades de expansión, la existencia de factores como incompatibilidad de proyectos, tensiones sociales y deterioro ambiental hacen evidente la necesidad de analizar con detenimiento las características específicas del desarrollo local en Valle de Bravo.

Es necesario destacar la confrontación social que prevalece como resultado del proceso de asentamiento de nuevos poblado- 
res, que paulatinamente han desplazado a los habitantes locales en las esferas sociales y económicas, dando lugar a una sociedad local dividida en la que los intereses y objetivos de sus integrantes se enfrentan de manera constante.

La evidente contraposición de las iniciativas locales identificadas en Valle de Bravo -en especial en torno a dos visiones diferentes para el aprovechamiento y protección del territorio y sus recursos naturales, que son su principal atractivo y fuente de recursos-impide la conformación de un proyecto común que aglutine el potencial y capacidades manifiestas de los actores locales.

Aunado a lo anterior, también es importante considerar que en este municipio se observan en algunos sectores de la población costumbres y estándares de vida de carácter internacional, derivados de la presencia de quienes aunque radican de manera definitiva en la Ciudad de México, o en alguna otra gran ciudad del país, poseen alguna casa de fin de semana en Valle de Bravo.

$\mathrm{Al}$ respecto, cabe señalar que aunque los productos y servicios que se generan en el municipio están orientados hacia el mercado interno más que al internacional (sólo $0.8 \%$ de turismo que visita el municipio proviene de otros países), deben cumplir con la calidad de los establecimientos que se ubican en el Distrito Federal, y aun en el extranjero, pues quienes los consumen o tienen acceso a tales bienes, tienen esas preferencias y estándares.

De este modo, en el municipio se observa que el contexto de la globalización está determinado en lo fundamental por los efectos que genera la presencia de consumidores nacionales, pero habituados a estándares internacionales de calidad en materia de servicios y productos de consumo inmediato, y aunque evidentemente éstos no son de acceso generalizado para la población local, la demanda ha dado lugar a que los proveedores locales estén en condiciones de afrontarla.

En este contexto, la valoración de las capacidades de los actores locales permitió identificar dos organizaciones paralelas de actores locales, mutuamente excluyentes, en las que los objetivos y proyectos comunes son diferentes, e incluso, opuestos.

La incompatibilidad de proyectos y visiones complica determinar una organización general de los actores locales y, por tanto, de un proyecto común. Lo anterior implica, en el caso de la organización, la existencia de liderazgos con capacidad de convocatoria y reconocimiento de la sociedad local, en el segmento que corresponde a cada una de las dos partes identificadas. Se considera que precisamente esta divergencia, o contraposición, de visiones y ob- 
jetivos le da un carácter polarizado a la capacidad organizacional de los actores locales de Valle de Bravo.

Esta situación subyace en la valoración de las capacidades instrumental y sistémica de los actores locales de este municipio, pues aunque durante nuestro trabajo de campo identificamos elementos que confirman su existencia para cada una de las dos organizaciones generales referidas, precisamente esa situación de sociedades paralelas induce su caracterización en términos de parcialidad.

La caracterización de las capacidades de los actores locales analizadas en el caso de Valle de Bravo, indica que éstas no se encuentran estructuradas en torno a objetivos y proyectos comunes, sino alrededor de dos posiciones contrapuestas, aparentemente irreconciliables, que evitan su aprovechamiento en aras de un proceso integral de desarrollo local que promueva la transformación cualitativa y sostenida de las condiciones de vida de la sociedad local y de sus expectativas de progreso.

\subsection{Villa Guerrero}

El municipio de Villa Guerrero se localiza en la zona sur del Estado de México. En el año 2005 contaba con una población estimada en más de 54,000 habitantes. Tiene una superficie de 20,773 hectáreas, de las cuales $53.28 \%$ son de uso forestal; 42.10\%, agropecuario y 4.62, urbano.

Su ubicación le proporciona una serie de características climáticas favorables para el desarrollo de la floricultura, factor que influyó de manera determinante en la realización de esta actividad de manera empresarial a partir de las décadas de los años cincuenta y sesenta del siglo pasado.

La preeminencia de las actividades primarias como base de la economía municipal prevalece hasta nuestros días y tiene tal relevancia que se constituye en un caso de referencia obligado en materia de análisis del desarrollo local, por el progreso que ha detonado en Villa Guerrero. La distribución de la PEA por sector hace evidente el hecho de que, en sentido contrario a la distribución estatal de la misma, en este municipio predominan las actividades primarias.

Esta característica -singular en el Estado de México- aporta evidencias empíricas que contradicen la creencia generalizada acerca de la poca rentabilidad de las actividades en el sector primario, pues la floricultura no sólo genera empleos directos en el 
Cuadro 7

Distribución de la PEA por sector

\begin{tabular}{cccc}
\hline Año & Primario & $\begin{array}{c}\text { Secundario } \\
\text { Porcentaje }\end{array}$ & Terciario \\
\hline 1960 & 87.61 & 3.70 & 8.69 \\
1970 & 78.20 & 4.30 & 17.50 \\
1980 & 78.76 & 5.82 & 15.42 \\
1990 & 81.25 & 5.63 & 13.12 \\
2000 & 74.4 & 6.40 & 19.20 \\
\hline
\end{tabular}

Fuente: Elaboración propia con base en datos del INEGI, Censos Generales de Población y Vivienda, 2000, 1990, 1980, 1970 y 1960.

municipio, sino que ha trascendido las fronteras nacionales ocupando mano de obra de otros países. ${ }^{4}$

En términos socioeconómicos, en Villa Guerrero se tiene un grado de marginación que se considera medio, cuyos indicadores se aprecian en el cuadro 8 .

En cuanto a su índice de desarrollo humano, Villa Guerrero se considera dentro de los municipios que tiene un grado medio alto; ocupa el décimo lugar regional en cuanto al índice de marginación. Al respecto, el cuadro 9 proporciona información más amplia en cuanto a la evolución del municipio en términos de su grado de marginación en el contexto nacional.

Un dato que resulta interesante es el de la emigración, pues aunque Villa Guerrero se ubica en una región que se distingue por el fenómeno cultural y económico del bracerismo, ${ }^{5}$ su porcentaje de emigración es muy bajo, pues se estima en aproximadamente 321 personas al año, que representa $0.63 \%$ de la población. Esto resulta de suma importancia en la definición y caracterización del desarrollo local de Villa Guerrero, pues posee una dinámica propia que le permite, entre otras cosas, generar los suficientes incentivos en el territorio municipal para tener un índice de desempleo de sólo $0.32 \%$, así como una emigración de $0.63 \%$ anual,

\footnotetext{
${ }^{4}$ Según información proporcionada por un funcionario del Consejo Mexicano de la Flor, con sede en Villa Guerrero, entrevistado en mayo de 2004, algunas empresas, fundamentalmente de capital japonés, cuentan con un sistema establecido de captación de mano de obra en estados como Chiapas y Oaxaca, y en países como Ecuador y Guatemala, desde donde se traslada la mano de obra que se utiliza en los invernaderos durante todo el año.

${ }^{5}$ El bracerismo es la emigración temporal, y en ocasiones definitiva, de hombres jóvenes hacia algunas poblaciones de los Estados Unidos, donde se emplean como peones o jornaleros y desde donde hacen llegar dinero a las familias que dejan en sus lugares de origen.
} 


\section{Cuadro 8}

Indicadores de marginación, 2002

\begin{tabular}{lc}
\hline \multicolumn{1}{c}{ Indicadores } & $\begin{array}{c}\text { Villa Guerrero } \\
\text { (porcentaje) }\end{array}$ \\
\hline Población & 50,829 \\
Población analfabeta mayor de 16 años & 12.65 \\
Población sin primaria completa mayor de 16 años & 45.69 \\
Ocupantes de viviendas sin drenaje ni excusado & 22.91 \\
Ocupantes de viviendas sin energía eléctrica & 4.19 \\
Ocupantes de viviendas sin agua entubada & 13.99 \\
Viviendas con hacinamiento & 59.17 \\
Ocupantes en viviendas con piso de tierra & 28.86 \\
Población en localidades con menos de 6,000 habitantes & 83.62 \\
Población ocupada con ingreso menor a dos salarios mínimos. & 70.93 \\
Índice de marginación & 0.714 \\
Grado & Alto \\
\hline
\end{tabular}

Fuente: Consejo Estatal de Población, Estado de México, Índices de marginación 2000. 124 municipios, 2002.

\section{Cuadro 9}

Comparativo del índice y grado de marginación, 1980-2000

\begin{tabular}{lrrrr}
\hline \multicolumn{1}{c}{ Año/aspecto a considerar } & 1980 & 1990 & 1995 & 2000 \\
\hline Índice de marginación & -0.780 & -0.073 & -0.185 & -0.124 \\
Grado de marginación & Alta & Media & Media & Media \\
Posición en el contexto nacional & 1,629 & 1,259 & & 1,304 \\
\hline
\end{tabular}

Fuente: Instituto Nacional para el Federalismo y el Desarrollo Municipal. Sistema Nacional de Información Municipal.

que como se verá más adelante, es la más baja en relación con los otros dos casos de estudio seleccionados.

De la caracterización territorial y socioeconómica de Villa Guerrero destacan, para efectos de la presente investigación, los siguientes aspectos:

- En Villa Guerrero se mantiene la vocación económica del territorio, teniendo como actividades principales las relacionadas con el cultivo de la tierra.

- A pesar de que se encuentra en una región caracterizada por altos índices de marginalidad, en el municipio se observa un bajo nivel de desempleo y de emigración, como resultado de la dinámica laboral generada en torno a la floricultura. 
- La floricultura ha producido una serie de efectos multiplicadores en los otros sectores de la economía, donde se advierte la proliferación de negocios y actividades industriales relacionadas con la principal actividad económica del municipio.

- No obstante lo anterior, los altos índices de analfabetismo y los bajos niveles de ingreso de los habitantes del municipio contrastan con la dinámica económica del mismo.

En este municipio se identificó una organización general dinámica de los actores locales, con objetivos claros y proyectos definidos en el contexto de la globalización, impulsados de manera decisiva por líderes con gran capacidad de convocatoria y reconocimiento de la sociedad local.

Dicha organización, impulsada por los liderazgos referidos, generó el posicionamiento de los floricultores de Villa Guerrero a la vanguardia de la producción florícola nacional, encabezando los organismos cúpula del sector y dio lugar a una relación de complementariedad entre los actores locales del municipio.

En este caso de estudio cabe señalar la capacidad instrumental de los actores locales, que va desde la concertación y el cabildeo de alto nivel con autoridades federales y estatales, hasta la realización de eventos de promoción local, regional y nacional en los que participan los pequeños productores, apoyados por los grandes productores de manera solidaria.

En términos de la capacidad sistémica de los actores locales de Villa Guerrero, 90\% de la producción florícola nacional que se exporta proviene de los invernaderos de este municipio, y en especial de los que localmente se consideran grandes productores, quienes cuentan con el conocimiento de la dinámica de los mercados internacionales, con vínculos específicos de utilidad para la promoción de su desarrollo y tienen acceso a tecnología de vanguardia para la promoción y realización de transacciones relacionadas con su actividad económica, que redundan en elementos positivos en cuanto al proceso de desarrollo local en el municipio.

No obstante lo anterior, un elemento a considerar en cuanto a la connotación de integralidad del proceso de desarrollo local de Villa Guerrero, es el que corresponde a la sostenibilidad, pues durante el trabajo de campo se observó que, en aras de incrementar la productividad y la calidad, se utilizan fertilizantes, plaguicidas y herbicidas sin un control regular por parte de las autoridades locales. 
Otra asignatura pendiente en este caso de estudio es el reflejo de los indicadores del tipo de desarrollo local articulado en mejores condiciones de vida para la población, puesto que indicadores como el grado de analfabetismo y los bajos niveles de ingresos de los trabajadores contrastan con las capacidades y la articulación de iniciativas, objetivos y estrategias observadas en este municipio.

\section{Conclusiones}

En la búsqueda del desarrollo local resulta fundamental la capacidad de organización de los actores en torno a un objetivo y proyecto común, pues la agregación de esfuerzos y recursos en este sentido permite elaborar e instrumentar estrategias para el aprovechamiento de los recursos endógenos y da cohesión y sentido a las iniciativas locales. La distinción de los actores del desarrollo local es importante, pues la preeminencia de las decisiones e iniciativas de los actores locales sobre las de actores externos, pero con incidencia en el ámbito local, da continuidad y viabilidad a los proyectos de desarrollo.

El contexto de la globalización es un elemento insoslayable en la búsqueda del desarrollo local, pues la incidencia de los aspectos estructurales de la misma genera cambios en la composición del factor de cohesión interna, trastocando así las relaciones sociales y económicas en los ámbitos locales.

La globalización como contexto para el desarrollo local se presenta de formas concretas, a través de elementos que afectan de manera diferenciada a las localidades:

- En San Mateo Atenco se manifiesta en los cambios generados en las preferencias de los consumidores, lo que da lugar al desplazamiento paulatino del producto local por el de procedencia externa y enfrenta a los productores locales a la necesidad de reestructurar sus procesos productivos para mantener su posición en el mercado interno.

- En Valle de Bravo el turismo es una actividad directamente vinculada con el mercado internacional, sin embargo, el nexo de este municipio con el contexto de la globalización no se observa en este sentido, sino en las modificaciones generadas en el factor de cohesión interna por parte del turismo residente a través de la generación de estándares de consumo de calidad internacional, así como en el plan- 
teamiento de estrategias de desarrollo propuestas por los actores locales.

- En Villa Guerrero la vinculación con el contexto global se manifiesta en función de la organización interna de los actores locales tanto en la producción como en la distribución de la flor. Elementos estructurales de la globalización como la consideración del conocimiento como fuerza productiva, la generación de innovaciones y de tecnología propia, así como la unión de la planta productiva con las instituciones de educación superior, hacen evidente la capacidad sistémica de los actores locales.

De lo anterior se infiere que la búsqueda del desarrollo local en el contexto global implica la valorización del ámbito local, más que la subordinación del mismo a las exigencias del primero. Implica la procuración de estándares internacionales de calidad y precio en los productos y servicios locales, aun cuando el destino de éstos sea el mercado interno.

Si bien el objetivo de esta investigación no fue realizar comparaciones entre realidades evidentemente diferentes, sí es posible hacer algunos señalamientos en relación con el resultado observado en la conjugación de las diferentes capacidades de los actores locales en cada municipio estudiado.

De este modo, se puede señalar que la capacidad organizacional de los actores locales resulta determinante en cuanto al impulso y enfoque de los procesos de desarrollo local revisados, lo cual a su vez se encuentra condicionado por el marco institucional que prevalece en cada localidad, así como por factores de índole sociocultural, económica y, por supuesto, de las externalidades relacionadas con el proceso de globalización.

En el cuadro 10 se muestra cómo visualizamos la estrategia de los actores en los procesos de desarrollo local estudiados.

Al respecto, el papel de las capacidades que dan lugar al desarrollo local es fundamental y tienen una connotación específica: la organizacional depende del marco institucional; la instrumental y sistémica dependen de la calificación de los actores locales. En todo caso, se puede señalar que el marco institucional local incide de manera determinante en los procesos de desarrollo local. 


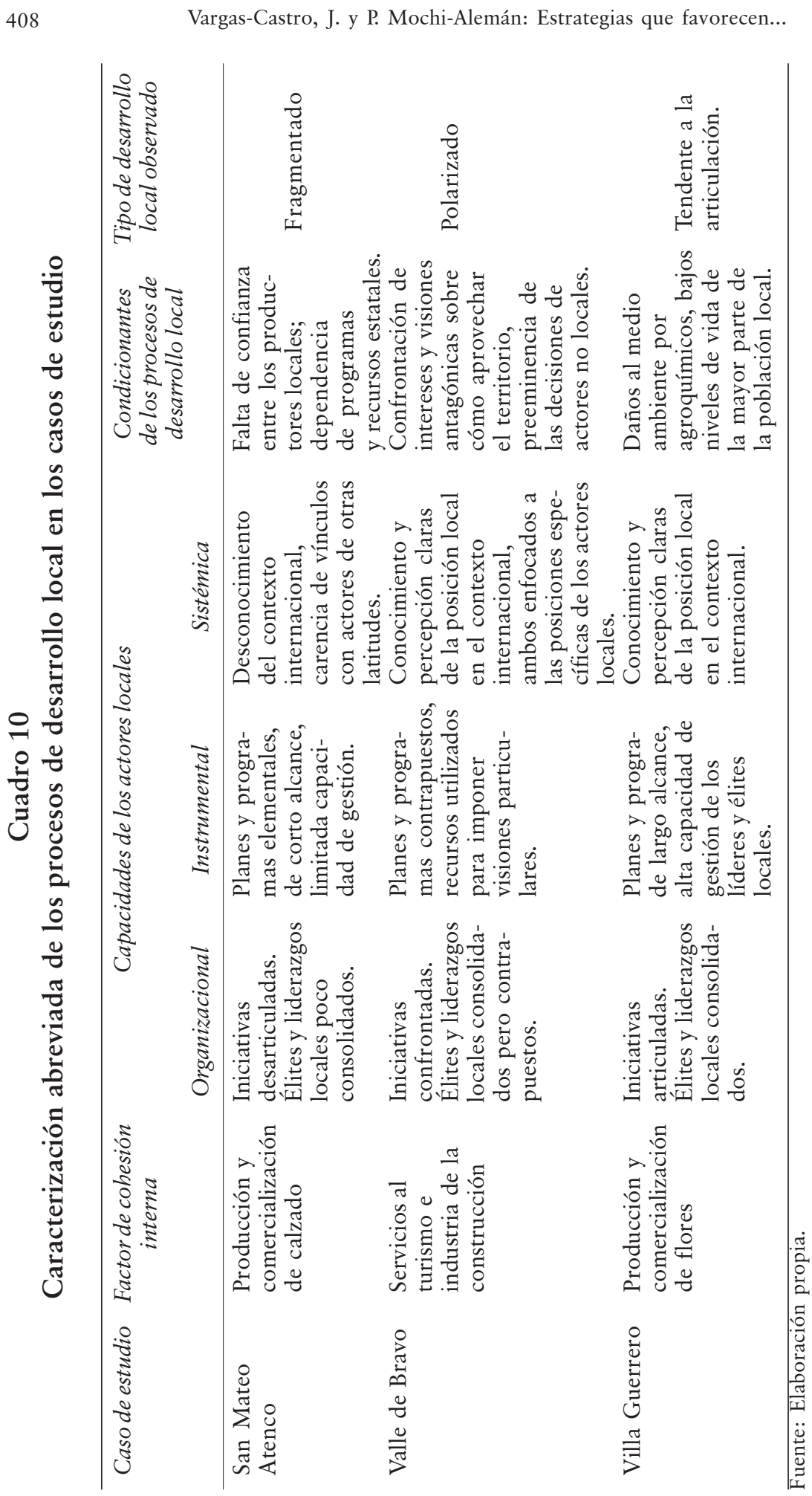

est26_4.pmd 408

27/05/2008, 03:49 p.m. 


\section{Bibliografía}

Albuquerque-Llorens, Francisco (1997), Cambio estructural, globalización y desarrollo económico local, CEPAL-ILPES, Santiago de Chile.

Albuquerque-Llorens, Francisco (2003), Desarrollo económico local, Centro Internacional de Formación de la orT, Turín.

Albuquerque-Llorens, Francisco (2005), "Las iniciativas locales de desarrollo y el ajuste estructural”, en Andrés Solarí Vicente y Jorge Martínez Aparicio (comps.), Desarrollo local, textos cardinales, Facultad de Economía, Universidad Michoacana de San Nicolás de Hidalgo, Morelia, México, pp. 69-89.

Axford, Barrie (1995), The global system, St. Martin Press, Nueva York.

Beck, Ulrich (1998), La sociedad del riesgo, Paidós, Barcelona.

Boisier, Sergio (2005), “Desarrollo local. ¿De qué estamos hablando? en Antonio Vázquez Barquero y Oscar Madoery (comps.), Transformaciones globales, instituciones y politicas de desarrollo local, Homosapiens Ediciones, Rosario, $119 \mathrm{p}$.

Castells, Manuel (1998), Local y global. La gestión de las ciudades en la era de la información, Taurus, España.

Consejo Estatal de Población, Estado de México (2002), Índices de marginación 2000. 124 municipios, Gobierno del Estado de México, México.

Coraggio, José Luis (2005), "La política urbana metropolitana frente a la globalización”, en Andrés Solarí Vicente y Jorge Martínez Aparicio (comps.) Desarrollo local, textos cardinales, Facultad de Economía, Universidad Michoacana de San Nicolás de Hidalgo, Morelia, México, pp. 222-252.

Dabat, Alejandro (2000), La globalización, el capitalismo informático-global y la nueva configuración espacial del mundo, CRIM-unam, Cuernavaca, México. 
Dabat, Alejandro (2002), "Globalización, capitalismo actual y nueva configuración espacial del mundo", en J. Basave et al., Globalización y alternativas incluyentes para el siglo XXI, Porrúa, México.

Dicken, Peter (1998), Transforming the World economy, Global shift, The Guilford Press, Nueva York.

Featherstone, Mike (1990), Global culture. Nationalism, globalization and modernity, Sage, Londres.

Garófoli, Gioachino (1995), "Desarrollo económico, organización de la producción y territorio", en A. Vázquez Barquero y Gioachino Garófoli (eds.) Desarrollo económico local en Europa, Colegio de Economistas de Madrid, Madrid, pp. 235-297.

Gereffi, Gary (1995), "Global production systems and third World development", en B. Satalling (ed.), Global change, regional response: the new international context of development, Cambridge University Press, Nueva York.

Gregersen N. y L. Johnson (1996), "Learning economies, innovation systems and European integration", Regional Studies, 31, Pergamon Press, Oxford, Reino Unido, pp. 479-490.

Harvey, David (1995), "Globalization in question. Rethinking Marxism” 8(4), University of California Press, Berkeley y Los Ángeles, pp. 1-17.

Lundavall, Bengt-Ake (1992), National system of innovation: toward a theory of innovation and interactive learning, Printer Publisher, Londres.

Madoery, Oscar (2001), "El valor de la política de desarrollo local”, en Antonio Vázquez Barquero y Oscar Madoery (comps.), Transformaciones globales, instituciones y politicas de desarrollo local, Homosapiens Ediciones, Rosario, pp. 200-229.

Mochi-Alemán, Prudencio y Cristina Girardo-Pierdominici (1998), "El desarrollo local como estrategia de los acto- 
res sociales”, Sociedad Civil, III(7), Demos, México, pp. 195-210.

Nonaka, Ikujiro e Hirotaka Takeuchi (1995), The knowledge-creating company, Oxford University Press, Nueva York.

OCDE (Organización para la Cooperación y el Desarrollo Económico) (2002), Mejores prácticas de desarrollo Local LEED, Cuaderno de trabajo 27, Correo de la unEsco, México.

Petrella, Ricardo (1992), “Three Analices of Globalization of Technology and R\&D”, Technology Analisis and Estrategic Management, 1 (4).

Polanyi, Karl (1966), The tacit dimension, Routledge \& Kegan Paul Ltd, Londres.

Scott, Allen John (1998), Regions and the World economy, Oxford University Press, Oxford.

Sen, Amartya (2000), "Desarrollo y libertad”, Planeta, México.

Vázquez-Barquero, Antonio (2001), “Desarrollo endógeno y globalización”, en Antonio Vázquez Barquero y Oscar Madoery (comps.), Transformaciones globales, instituciones y politicas de desarrollo local, Homosapiens, Rosario, pp. 76-99.

Vázquez-Barquero, Antonio (2005), "Desarrollo económico local y descentralización”, en Andrés Solarí Vicente y Jorge Martínez Aparicio (comps.), Desarrollo local, textos cardinales, Facultad de Economía, Universidad Michoacana de San Nicolás de Hidalgo, Morelia, México, pp. 33-68.

Torres-Noyola, Francisco (2002), Programa fundamental para el desarrollo económico del Estado de México hacia el 2005 $y$ de competitividad visión 2020. (Cluster de calzado), ITESM, México.

Vargas-Castro, José Alejandro (2006), El desarrollo local en el contexto de la globalización. Tres casos de estudio en el Estado de México: San Mateo Atenco, Valle de Bravo y Villa Guerrero, INAP-UAEM-GEM, México. 
Yoguel, Gabriel (2005), "Creación de competencias en ambientes locales y redes productivas", en Andrés Solarí Vicente y Jorge Martínez Aparicio (comps.), Desarrollo local, textos cardinales, Facultad de Economía, Universidad Michoacana de San Nicolás de Hidalgo, Morelia, México, pp. $155-175$.

Zack (1999), “Developing a Knowledge Strategy", Management Review, California, 41 (3), pp. 125-145.

Recibido: 10 de agosto de 2006. Reenviado: 11 de diciembre de 2006. Aceptado: 2 de enero de 2007.

José Alejandro Vargas Castro. Es doctor en ciencias sociales por El Colegio Mexiquense. Actualmente labora en la Facultad de Arquitectura y Diseño de la Universidad Autónoma del Estado de México (UAEM). Su línea de investigación se centra en: desarrollo local, desarrollo urbano, municipios y descentralización. Entre sus publicaciones destacan: El desarrollo local en el contexto de la globalización. Tres casos de estudio en el Estado de México: San Mateo Atenco; Valle de Bravo y Villa Guerrero, México, INAP-UAEM-GEM (2006); "Las nuevas teorías del desarrollo y el desarrollo local", Páramo del Campo y la Ciudad, Órgano del Centro de Estudios sobre Marginación y Pobreza del Estado de México, 10, pp. 3-11 (2006); "La participación social en la planeación del desarrollo urbano municipal: retos y perspectivas para el Estado de México", Legado de Arquitectura y Diseño, Universidad Autónoma del Estado de México, México, 3, pp. 21-36 (2006).

Prudencio Óscar Mochi Alemán. Es doctor en estudios latinoamericanos por la Universidad Nacional Autónoma de México (UNAM). Actualmente se encuentra adscrito al Centro Regional de Investigaciones Multidisciplinarias (UNAM). Su línea de investigación se centra en: globalización y desarrollo local, tecnologías de información y comunicación, la industria del software en México. Entre sus publicaciones destacan: La industria del software en México en el contexto internacional y latinoamericano, México, CRIM-UNAM (2006); "Las formas de organización, los procesos de calidad y los trabajadores del conocimiento en la indus- 
tria del software", en Carlos Massé Narváez (coord.), La complejidad de las ciencias sociales en la sociedad de la información y la economía del conocimiento, Toluca, El Colegio Mexiquense. pp. 239-272 (2005), "Las tecnologías de la información y la comunicación como herramientas para el desarrollo", en Karla Valverde y Alejandra Salas Porras (coords.), El desarrollo. Diversas perspectivas en las ciencias, las instituciones, el Estado, la democracia, la cultura, la sociedad civil, México, Gernika, pp. 379410 (2005). 